\title{
Bilateral Epidural Hematoma: Case Illustration and Classification Proposal
}

\author{
${ }^{1}$ Miguel AM Morán, ${ }^{2}$ Sebastian Toro, ${ }^{3}$ Claudia Restrepo, ${ }^{4}$ Juan E Muñoz, ${ }^{5}$ Erik Muñoz
}

\section{RESUMEN}

El hematoma epidural es una complicación frecuente del trauma craneoencefálico cerrado, sin embargo, es infrecuente la presentación de manera bilateral y se asocia a elevada morbimortalidad. Presentamos un caso de paciente masculino de 28 años victima de accidente de transito quien presenta hematoma epidural inicialmente unilateral, y que después de 4 días se convierte en un hematoma epidural bilateral.

Palabras Clave: Bilateral, Craneoencefálico, Epidural, Hematoma, Trauma.

How to cite this article: Morán MAM, Toro S, Restrepo C, Muñoz JE, Muñoz E. Bilateral Epidural Hematoma: Case Illustration and Classification Proposal. Panam J Trauma Crit Care Emerg Surg 2017;6(2):127-130.

Source of support: Nil

Conflict of interest: None

\section{ABSTRACT}

Epidural hematoma is a frequent complication of closed craneoencephalic trauma, however, bilateral presentation is nonfrequent and it is associated with high morbimortality. We report a case of a male patient who suffered a road traffic accident and has unilateral epidural hematoma, which, after 4 days, becomes bilateral epidural hematoma.

Keywords: Bilateral, Craneoencephalic, Epidural, Hematoma, Trauma.

\section{INTRODUCCIÓN}

El trauma es una de las causas más frecuentes de muerte y discapacidad en la población de 12 a 45 años, para el año 2012 el politraumatismo (incluyendo el trauma cráneo encefálico) correspondió al 65.5\% de las lesiones fatales en accidente de tránsito, seguido del TCE aislado en un $27.2 \% .^{1}$ Los hematomas epidurales (también llamados extradurales) se presentan en el $2 \%$ de todos los casos de TCE y en más del 15\% de las injurias de cabeza

\footnotetext{
${ }^{1}$ Intern, ${ }^{2-4}$ Resident, ${ }^{5}$ Professor

${ }^{1-5}$ Department of Neurosurgery, Hospital Militar Central Universidad Militar Nueva Granada, Bogotá, Colombia

Corresponding Author: Miguel AM Morán, Intern, Department of Neurosurgery, Hospital Militar Central, Universidad Militar Nueva Granada, Bogotá, Colombia, Phone: +5713486868 e-mail: miguel.mamoran@gmail.com
}

fatales. ${ }^{2}$ La presentación usual del hematoma epidural es unilateral y habitualmente se ubica en región temporal y parietal, sin embargo, en un $2 \%$ de todos los casos de hematomas epidurales puede ser de presentación bilateral ${ }^{3}$ lo cual aumenta de manera drástica la mortalidad $(>30 \%) .{ }^{4}$ Presentamos el caso de un paciente que tras un accidente de tránsito presentó inicialmente hematoma epidural unilateral y después de 4 días presentó hematoma epidural bilateral, requiriendo procedimiento neuroquirúrgico.

\section{REPORTE DE CASO}

Se trata de un paciente masculino de 28 años quien ingresa a un servicio de urgencias en la ciudad de Arauca por presentar cuadro clínico consistente en trauma de cráneo posterior a accidente de transito en calidad de conductor de motocicleta, ingresando desorientado, somnoliento, refiriendo cefalea de moderada intensidad y con un Glasgow de 12/15, posteriormente presentó deterioro progresivo de su condición neurológica por lo cual realizaron intubación orotraqueal y solicitaron una TAC cerebral simple (Figura 1).

En la tomografía inicial realizada al paciente se evidencia hematoma epidural de aprox. 31,8cc de volumetría, por las condiciones clínicas, paraclínicas y por la no disponibilidad en el momento del servicio de Neurocirugía, se decidió manejo médico con medidas de neuroprotección en unidad de cuidado intensivo. Durante su estancia en UCI el paciente no presentó deterioro neurológico ni hemodinámico. Al cuarto día posterior al trauma deciden solicitar una TAC cerebral control (Figura 2), en la cual se evidencia hematoma epidural temporal izquierdo de características concordantes con el primer TAC y además encuentran hematoma epidural temporal derecho con volumetría de 18cc.

Debido a los hallazgos encontrados en el TAC control, deciden realizar remisión a nuestra institución. Recibimos al paciente bajo intubación orotraqueal, con apertura ocular espontánea y obedeciendo ordenes sencillas ECG $11 \mathrm{t} / 15$, por el alto riesgo de aumento de colección epidural y por consiguiente riesgo de deterioro neurológico se decidió llevar a craneotomía para drenaje de hematoma epidural bilateral, realizada esta en el servicio de neurocirugía del Hospital Militar Central. 

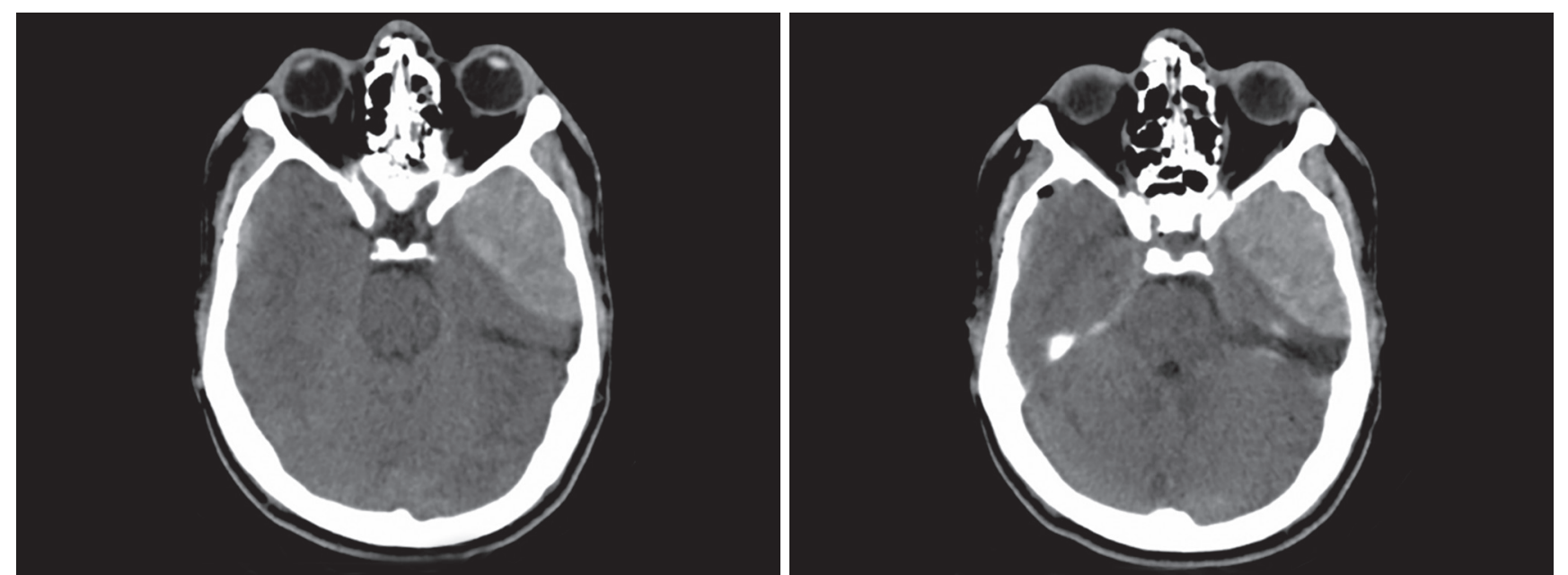

Figura 1: TAC Cerebral simple día 1 post trauma: Se evidencia hematoma epidural temporal izquierdo con volumetría de 31.8cc, hematoma epidural laminar temporal derecho, con neumoencéfalo y fractura panfacial asociada
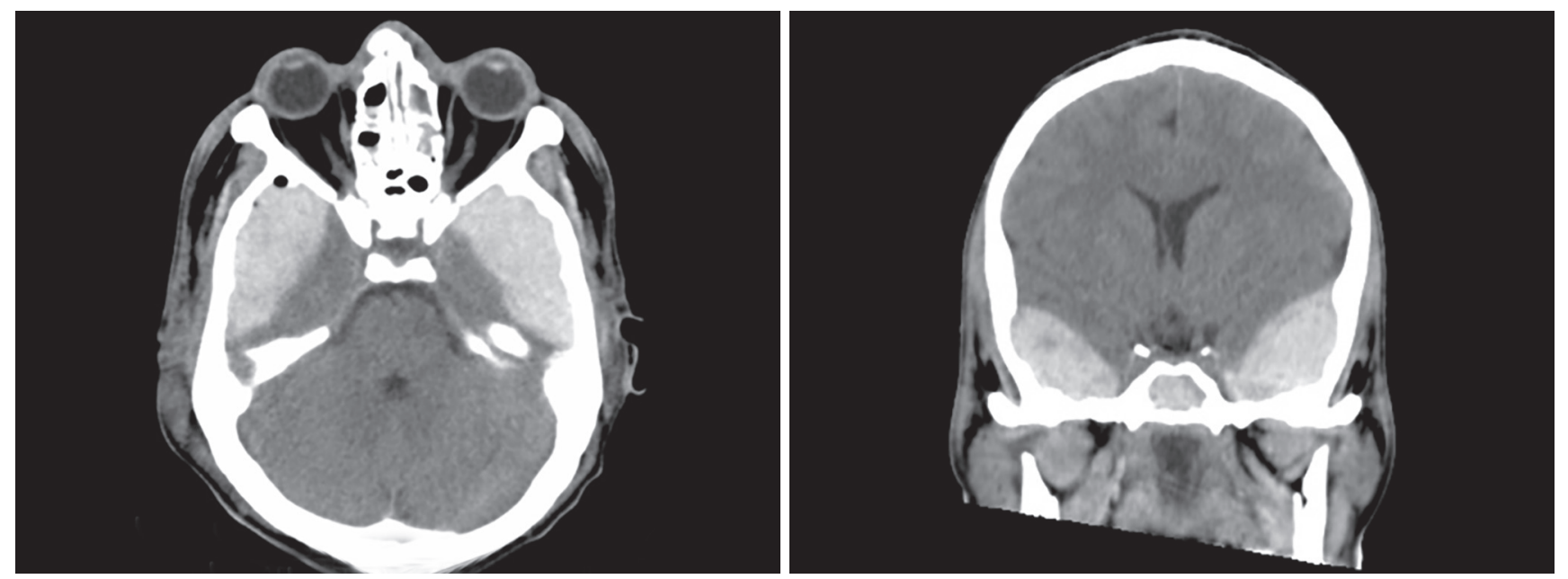

Figura 2: TAC cerebral 4to día posterior al trauma. Evidencia hematoma epidural temporal derecho de volumetría aprox $18 \mathrm{cc}$, Hematoma epidural izquierdo de características similares al estudio imagenológico previo
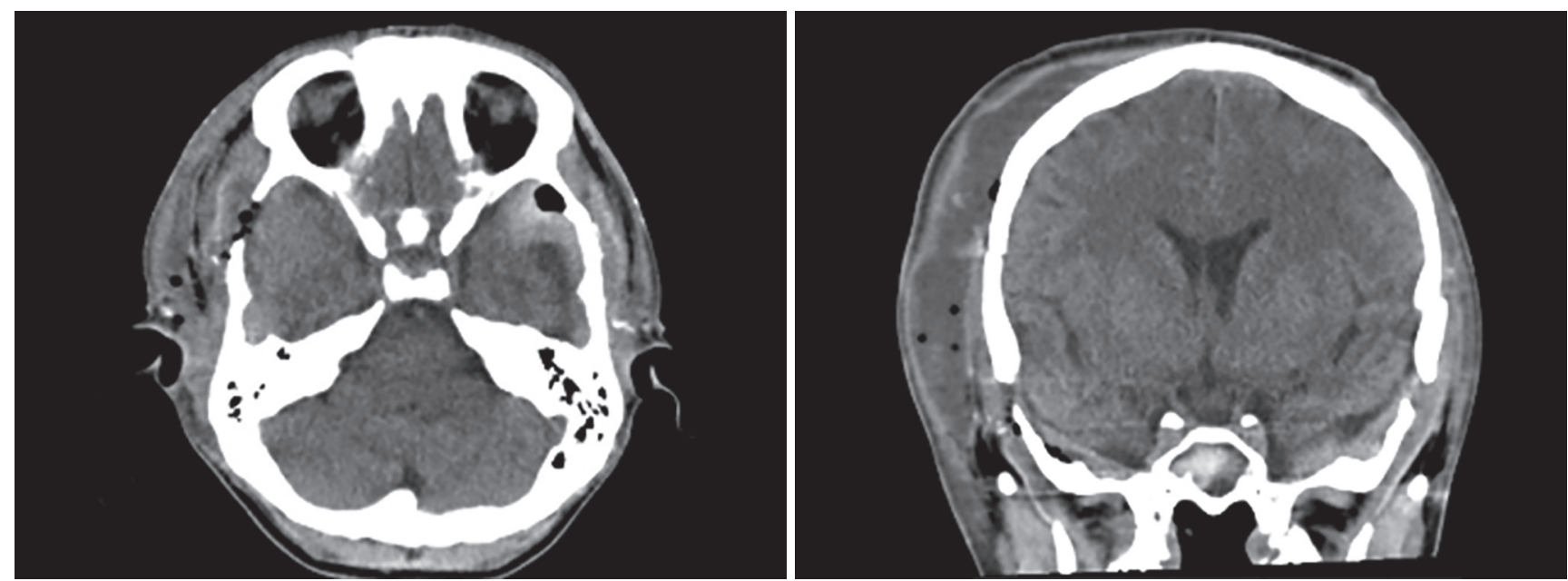

Figura 3: TAC cerebral posoperatoria. Sin evidencia de colección sanguínea. Edema de tejidos blandos derecho. Resto de hallazgos concordantes con neuroimágenes anteriores

Se realizó craneotomía descompresiva bilateral en un único tiempo quirúrgico, procedimiento sin complicaciones, posterior a la cirugía el paciente evolucionó de manera satisfactoria, y fue manejado medicamente.
Se tomaron neuroimágenes control evidenciando múltiples fracturas craneofaciales concordantes con hallazgos anteriores y edema de tejidos blandos derecho (Fig 3). Finalmente se dio egreso hospitalario al paciente, quien 
hasta el día de hoy no ha presentado complicaciones ni nueva sintomatología.

\section{DISCUSIÓN}

Porcentualmente los hematomas epidurales son mucho menos frecuentes que los hematomas subdurales, ocurren más en personas jóvenes y en hombres. Su presentación clínica "clásica" (que ocurre en menos del 27\% de los casos) se compone por perdida de la conciencia inmediatamente posterior al trauma, seguida de un intervalo lúcido del paciente durante algunas horas y posteriormente deterioro clínico progresivo ${ }^{5}$. Revisando la fisiopatología de estos encontramos que la sintomatología varía según el origen del sangrado; si la sangre es arterial la sintomatología es rápidamente progresiva o sigue el curso "clásico" anteriormente mencionado, llevando al paciente a Glasgow de bajo puntaje en poco tiempo, en cambio, cuando el origen del sangrado es venoso el paciente puede durar hasta días sin presentar deterioro y eventualmente se complicara una vez que el hematoma se forme, lo que en algunas referencias se encuentra como "hematoma epidural retrasado", al cual se le han atribuido algunos factores de riesgo como son: Descenso de PIC (sea osmótica o quirúrgicamente), coagulopatías y fractura craneal. ${ }^{5,6}$ Lo anterior podría explicar el caso de nuestro paciente (inicialmente único hematoma epidural izquierdo y posteriormente hematoma epidural retrasado derecho).

Cuando se hace la revisión de la literatura se encuentra que en reportes de caso anteriores clasifican los hematomas epidurales bilaterales de distintas formas, sin embargo, no existe una clasificación general de estos. Teniendo en cuenta lo anterior y con base en la bibliografía proponemos el siguiente modelo (Tabla 1): Según su tiempo de aparición; sincrónicos cuando en la primera TAC se evidencian los dos hematomas, asincrónicos cuando se evidencia primero un hematoma y posteriormente en una TAC control se evidencia un hematoma distinto. ${ }^{7}$ Según su posición a partir de la línea media; simétricos cuando por ejemplo los dos hematomas aparecen en las regiones temporales, asimétricos cuando por ejemplo aparece un hematoma temporal izquierdo y un hematoma

Tabla 1: Clasificación propuesta por los autores de los hematomas epidurales bilaterales

Según localización craneocaudal9

\begin{tabular}{|c|}
\hline \multicolumn{2}{|c|}{ Según localización craneocaudal9 } \\
\hline Supratentoriales \\
\hline \multicolumn{2}{|c|}{ Infratentoriales } \\
\hline Según posición frente a línea media8 \\
\hline Simétricos \\
\hline Según tiempo de aparición7 \\
\hline Asincrónicos
\end{tabular}

occipital derecho. ${ }^{8}$ Finalmente, según su localización craneocaudal; supratentoriales e infratentoriales, siendo estos últimos el $1.2 \%$ al $11 \%$ de todos los hematomas epidurales. ${ }^{9}$ Siendo entonces nuestro caso un hematoma epidural bilateral supratentorial simétrico asincrónico. En cuanto a la formación fisiopatológica de estos Frank et al. propusieron en 1982 que el origen de los hematomas epidurales bilaterales es debido a una única fuerza con vector anteroposterior que causa desprendimiento de la duramadre, ${ }^{10} \sin$ embargo, es probable que esta propuesta aplicaría más para los hematomas epidurales bilaterales frontales y subagudos, puesto que una divergencia en los vectores de fuerza al momento del trauma explicaría los de presentación asincrónica y/o asimétrica.

En cuanto al tratamiento siempre que se encuentre un hematoma epidural bilateral la craneotomía descompresiva debe ser realizada ${ }^{11}$, preferiblemente de manera bilateral, ya que esto reduce la posibilidad de un nuevo tiempo quirúrgico y se tienen buenos resultados ${ }^{12} \sin$ embargo, en muchas ocasiones esto no es posible ya sea por dificultad en el posicionamiento, o cuando existen hematomas asimétricos, o por no disponibilidad de dos neurocirujanos, motivo por el cual algunos autores han discutido acerca de cual hematoma se debe operar primero, si el hematoma de mayor volumen o el hematoma que se encuentre más cerca de áreas elocuentes, concluyendo que se debe operar primero el hematoma localizado en el hemisferio dominante si el volumen del otro no es tan grande. ${ }^{13}$ Por otra parte los hematomas epidurales bilaterales asincrónicos pueden o no requerir manejo quirúrgico, según el tamaño de la colección y la sintomatología y evolución del paciente

\section{CONCLUSIONES}

Los hematomas epidurales bilaterales son de rara presentación, tienen mayor morbimortalidad que los hematomas epidurales unilaterales y pueden ser clasificados según tiempo de aparición, posición en referencia a la línea media y si son supratentoriales o infratentoriales. El tratamiento quirúrgico está indicado en todos los hematomas epidurales bilaterales, preferiblemente debe realizarse cirugía bilateral, sin embargo, cuando esto no es posible, la elección de cual hematoma operar primero debe ser tomada por el neurocirujano encargado basado en su criterio y experiencia.

\section{BIBLIOGRAFÍA}

1. Colombia. Ministry of Health and Social Protection, Colciencias, MEDITECH Foundation. Clinical Practice Guide for Diagnosis and Treatment of Adults with Severe Cranioencephalic Trauma. [Ministerio de Salud y Protección Social, Colciencias, Fundación MEDITECH. Guía de práctica clínica para diagnóstico y tratamiento de adultos con trauma 
craneoencefálico severo.] SGSS - 2014 Guía No. 30 GPC-TCE. Bogotá, 2014. (Span)

2. Blumbergs, P. Neuropathology of traumatic brain injury. In: Youmans Neurological Surgery. 6th ed. Elsevier; 2011. pp. 3288-3299.

3. Gorgulu A, Cobanoglu S, Armagan S, Karabagli H, Tervuz M. Bilateral epidural hematoma. Neurosurg Rev 2000;23:30-33.

4. Huda MF, Mohanty S, Sharma V, Tiwari Y, Choudhary A, Singh VP. Double extradural hematoma: an analysis of 46 cases. Neurol India 2004;52 Dec(4):450-452.

5. Greenberg, M. Handbook of Neurosurgery. 7th ed. New York: Thieme; 2010.

6. Agrawal A. Bilateral symmetrical parietal extradural hematoma. J Surg Tech Case Report 2011;3:34-36.

7. Eftekhar B, Ketabchi E, Ghodsi M, Esmaaeli B. Bilateral asynchronous acute epidural hematoma: a case report. BMC Emergency Medicine 2003;3:1.
8. Patil AB, Patil NA. Bilateral extradural hematoma: a case report. IJSS Journal of Surgery 2015;1:16-17.

9. Karasu A, Sabanci PA, Izgi N, Imer M, Sencer A, Cansever T, Canbolat A.. Traumatic epidural hematomas of the posterior cranial fossa. Surg Neurolo 2008 Mar;69(3):247-251.

10. Frank E, Berger TS, Tew JM Jr. Bilateral epidural hematomas. Surg Neurol 1982 Mar;17(3):218-222.

11. Pereira C, Dutra A, Andrade M. Hematoma extradural bilateral: Considerations on four cases. J Bras Neurocirurg 1997; 8(1):20-22.

12. Idei $M$, Shima $T$, Nishida $M$, Yamane $K$, Mihara $C$, Hatayama $T$, Onda H, Manabe K, Yokota A.. Symmetrical bilateral epidural hematoma after head injury in the mid parietooccipital region: case report. No Shinkei Geka 2004 Apr;32(4): 379-382.

13. Barlow P, Kohi YM. Acute simultaneous bilateral extradural hematoma. Surg Neurol 1985 Apr;23(4):411-413. 